\title{
Effect of Neuromuscular Electrical Stimulation (NMES) on swallowing in Persons with Parkinson's disease exhibiting Dysphagia: A Systematic Review
}

M Sonam Belliappa', SP Goswami²

${ }^{1}$ Junior Research Fellow (JRF), Department of Speech Language Pathology/All India Institute of Speech and Hearing (AIISH), Mysuru, India. ${ }^{2}$ Head of the Department (HOD), Professor of Speech Pathology, Department of Speech Language Pathology/All India Institute of Speech and Hearing (AIISH), Mysuru, India.

*Corresponding author: M Sonam Belliappa, Junior Research Fellow (JRF), Department of Speech Language Pathology/All India Institute of Speech and Hearing (AIISH), Mysuru, India.

Received date: January 06, 2020; Accepted date: March 11, 2021; Published date: March 15, 2021

Citation: MS Belliappa, SP Goswami. (2021) Effect of Neuromuscular Electrical Stimulation (NMES) on swallowing in Persons with Parkinson's disease exhibiting Dysphagia: A Systematic Review. International Journal of Clinical Case Reports and Reviews. 6(4); DOI: 10.31579/2690$4861 / 105$

Copyright: ( 2021 M Sonam Belliappa, This is an open-access article distributed under the terms of the Creative Commons Attribution License, which permits unrestricted use, distribution, and reproduction in any medium, provided the original author and source are credited.

\begin{abstract}
:
Swallowing is an essential activity required for survival. The global definition of dysphagia is simply difficulty in swallowing. Around $80 \%$ of individuals with Parkinson's disease (PD) are diagnosed with dysphagia in any stage of their disease progression. Dysphagia therapy in these individuals has gained considerable importance in improving the quality of life in the recent past. With the advancement of technology related to dysphagia management, the use of Neuromuscular Electrical Stimulation (NMES) in dysphagia management is studied extensively, and the efficacy of it in successfully easing dysphagia symptoms in PD is still being evaluated. Hence this systematic review is aimed to give an overview regarding the current status of the literature available on the effect of NMES as a method of dysphagia rehabilitation on swallowing function in persons with PD. The present review will collate and critically analyse the existing knowledge of neuromuscular electrical stimulation in dysphagia management of persons with Parkinson's disease. The authors did a systematic literature search following the PRISMA guidelines using electronic databases for studies reporting the use or effect of NMES on swallow function, aiding management of dysphagia in persons with PD. The quality and the levels of evidence of articles included were in accordance with the ABC rating scale by Siwek et al [20]. 3 out of 38 publications identified met the inclusion criteria. The authors carried out a systematic, qualitative and critical analysis of the articles finalised. The summary of the reviewed articles revealed the dearth of evidence and several methodological flaws as well as highlighted the prevailing research gap in this arena. This review additionally aids Speech-Language and Swallow Pathologists in clinical decision making and future directions concerning selecting Neuromuscular Electrical Stimulation (NMES) as a treatment option for managing dysphagia in persons with PD.
\end{abstract}

Keywords: systematic review; parkinson's disease; dysphagia. Swallowing; dysphagia rehabilitation; dysphagia management; neuromuscular electrical stimulation

\section{Introduction}

Parkinson's disease (PD) is one of the most commonly occurring neurological disorders globally, with a prevalence of more than 10 million people living worldwide [7]. The incidence and prevalence of oropharyngeal dysphagia are relatively higher and affects more than 52$82 \%$ of persons with PD [4], [5]. Dysphagia often is asymptomatic in mildly impaired PD [15], [18] and can progress to become significantly debilitating as it is associated with neuronal degeneration and subsequent depletion of dopamine and its metabolites within the basal ganglia. Approximately around $80-95 \%$ of individuals with PD exhibit swallowing problems at any stage of disease progression [17], [11] and $30-45 \%$ die of aspiration pneumonia [24]. Bradykinesia, rigidity and tremors of the swallowing musculature frequently influence dysphagia in
$\mathrm{PD}$, which can interfere in the motor control of swallowing increasing the risk for penetration and laryngeal aspiration [16]. The physiological changes causing dysphagia is multifactorial, including cognitive impairment, head and neck posturing during meals, generalised upper extremity dysmotility and impulsive feeding behaviour [21]. Videofluroscopic Swallowing Studies (VFSS) studies report specific problems seen in all three stages of swallow including slow Pharyngeal Transit Time (PTT), Oral Transit Time (OTT), vallecular pooling and aspiration [2].

Early management of dysphagia is of utmost importance to prevent the complications including aspiration pneumonia, dehydration, malnutrition and low quality of life. Among the multidisciplinary team managing dysphagia in PD, Speech Language Pathologists (SLP) may be considered 
as the primary service provider and therefore needs to adopt a preventive, symptom-based management approach. Dysphagia is now being appreciated as a significant public health problem giving rise to several novel techniques and strategies which are currently being evaluated and may reduce the impact of dysphagia on the patient in future. Current literature on the management of dysphagia has evidenced positive effects of several treatment strategies (including consistency modification, Thermotactile Stimulation (TTS), Expiratory muscle strength training (EMST), Lee Silverman Voice Technique(LSVT) and Video-Assisted Swallow Therapy (VAST)) with an improved swallow safety in persons with PD [23].

Neuromuscular Electrical Stimulation (NMES) is one such novel noninvasive technological advancement in the management of dysphagia, currently being evaluated and may reduce the impact of dysphagia in persons with PD in future. The electrical stimulation can increase strength, range of motion and offset the effects of disuse. The rationale for the use of NMES in persons with PD was to enhance the contraction of the affected muscle, causing dysphagia without fatiguing the individual receiving it [2]. Several studies targeting dysphagia management in PD have been published with seldom articles, including NMES as one of the therapeutic method. The effect of NMES on swallowing in PD is yet to be explored, and further investigations concerning clinical and long term applicability is needed. This systematic review aimed to summarise and critically analyse the existing studies on the effect of NMES on swallowing in individuals with PD exhibiting dysphagia and to explore the effects of NMES in dysphagia management in PD.

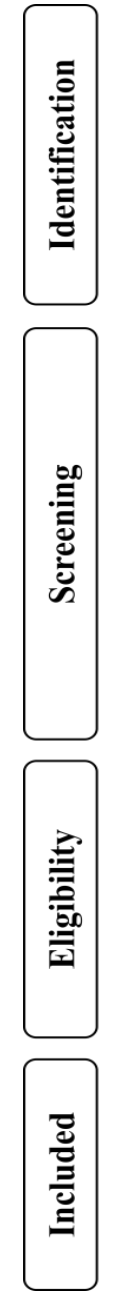

\section{Method}

Two reviewers independently carried out a systematic literature search following the PRISMA guidelines [14] for the articles reporting use of or effect of NMES on swallowing in persons with PD for a period after January 2000 to January 2020, using various electronic databases including google scholar, PubMed, Medline, Embase, Reference searches and website of the articles specific to the topic of interest (dysphagia journals, the dysphagia research society). The papers judged relevant by the reviewers were future assessed for inclusion. A discussion among the authors settled differences in their search.

The mesh terms used were swallowing or dysphagia combined with PD, NMES or electrical stimulation or vital stim combined with PD. To identify the recently published articles, free text words deglut*or swallow*or dysphg* or NMES* or vital stim therapy *or electrical stimulation* were combined with Parkinson's or parkinsonian disorders. After a first selection based on title and abstracts, a standard inclusion was made using original articles. A total of 18 articles were retrieved, of which only studies involving the use/effect of NMES in dysphagia management /swallowing of PD done by SLPs were included irrespectively. Review studies and meta-analysis, Grey literature, papers presented in conferences, Non-English language, duplicated studies, single case study, a study of NMES on voice and on wrong patient population or any other dysphagia therapeutic intervention in PD were excluded. To rate the quality of the evidence retrieved, the authors used the $\mathrm{ABC}$ rating scale by Siwek et al. The authors briefly described all the studies included, summarised and critically analysed each article.

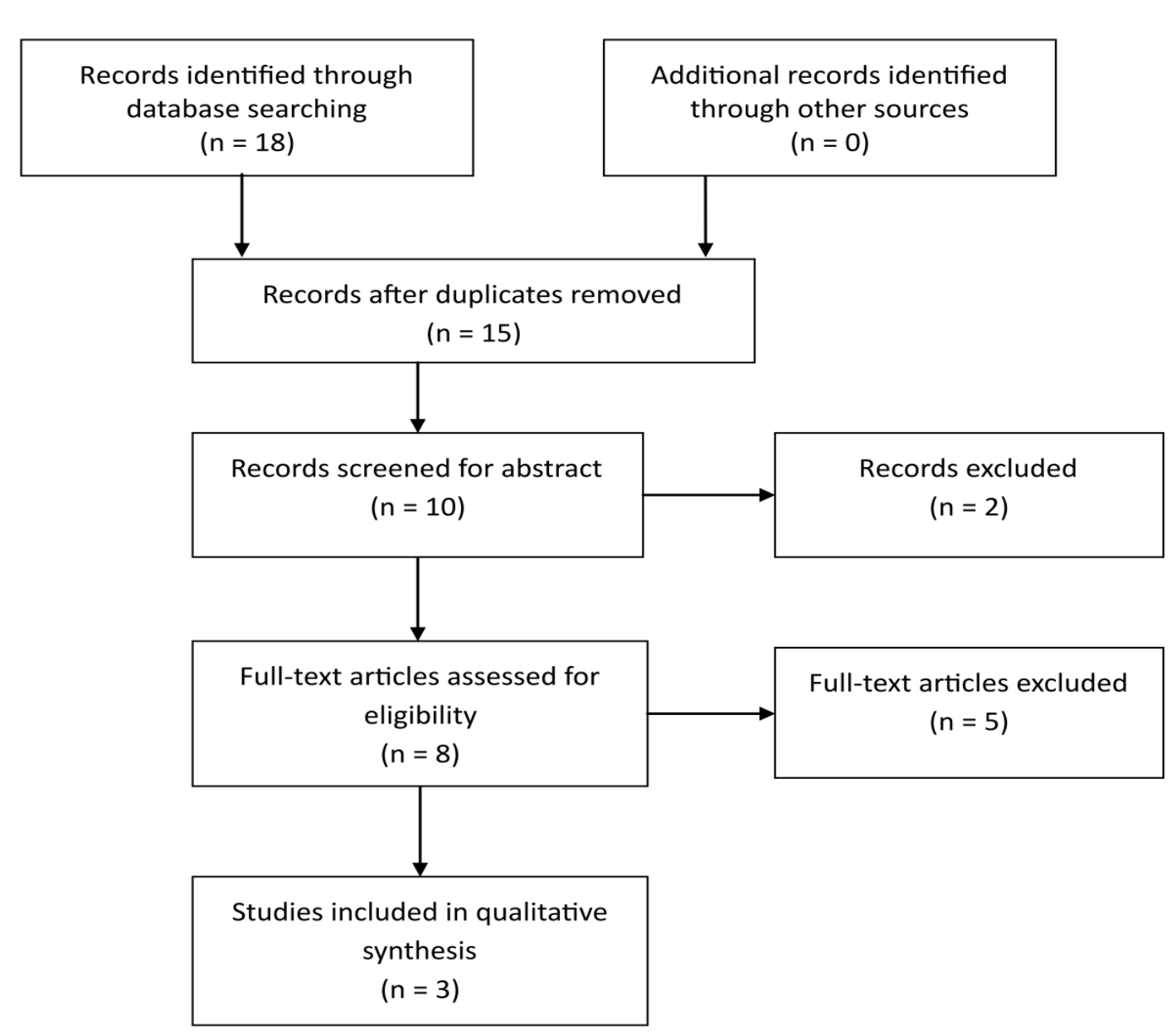




\section{Results}

The literature search retrieved 18 articles, of which only three studies were included after screening for inclusion, suggested a sheer of lack of evidence based research in the current area. The finalised articles are summarised in Table 1

\begin{tabular}{|c|c|c|c|c|c|c|c|c|c|}
\hline $\begin{array}{l}\text { S1 } \\
\text { no }\end{array}$ & Author & Year & Design & $\begin{array}{l}\text { Number of } \\
\text { subjects }\end{array}$ & Treatment protocol & Electrode placements & $\begin{array}{l}\text { Outcome measures / } \\
\text { instrumentation }\end{array}$ & Result & $\begin{array}{l}\text { Evidence } \\
\text { level }\end{array}$ \\
\hline 1 & $\begin{array}{l}\text { Heijne } \\
n \text { et al } \\
{[6]}\end{array}$ & 2012 & PPD & $\begin{array}{l}\text { PD with } \\
\text { Dysphagia } \\
n=88\end{array}$ & $\begin{array}{l}\text { Authors } \\
\text { between } 3 \text { groups (group } 1 \\
\text { received only traditional } \\
\text { swallow therapy; group } 2 \\
\text { received only NMES; and } \\
\text { group } 3 \text { received both } \\
\text { NMES +traditional } \\
\text { therapy). } \\
\text { NMES given at motor } \\
\text { level for } 13-15 \text { sessions of } \\
0.5 \text { hour per session }\end{array}$ & Suprahyoid muscles & $\begin{array}{l}\text { Quality of life using } \\
\text { Swallowing Quality } \\
\text { of Life, MD. } \\
\text { Anderson } \\
\text { Dysphagia } \\
\text { inventory, single } \\
\text { item dysphagia } \\
\text { severity scale } \\
\text { Functional Oral } \\
\text { Intake Scale scores } \\
\text { taken post } \\
\text { pre therapy por and after } 3 \\
\text { therapy and } \\
\text { months }\end{array}$ & 0 & $\begin{array}{l}\text { A } \\
(\mathrm{RCT})\end{array}$ \\
\hline 2. & $\begin{array}{l}\text { Baijens } \\
\text { et al [2] }\end{array}$ & 2012 & PPD & $\begin{array}{l}\text { PD with } \\
\text { Dysphagia } \\
n=10\end{array}$ & $\begin{array}{l}\text { Transcutaneous NMES } \\
\text { given for } 1 \text { session and } \\
\text { compared between } 3 \\
\text { groups with respect to } \\
\text { electrode placements. } \\
\text { group1: received } \\
\text { stimulation with electrode } \\
\text { placed on submental } \\
\text { region } \\
\text { (above the hyoid bone), } \\
\text { group } 2 \text { on pharyngeal } \\
\text { region } \\
\text { (below the hyoid bone) and } \\
\text { group } 3 \text { received with } \\
\text { electrodes placed on } \\
\text { submental }+ \text { pharyngeal } \\
\text { region with } 4 \text { electrodes } \\
\text { connected on each side of } \\
\text { the midline of the neck }\end{array}$ & $\begin{array}{l}\text { Submental and } \\
\text { pharyngeal region }\end{array}$ & $\begin{array}{l}\text { Videofluroscopic } \\
\text { Swallow Study } \\
\text { for temporal, spatial } \\
\text { and visoperceptual } \\
\text { variables }\end{array}$ & 0 & $\begin{array}{l}\mathrm{B} \\
(\mathrm{NRCT})\end{array}$ \\
\hline 3. & $\begin{array}{l}\text { Baijens } \\
\text { et al [3] }\end{array}$ & 2013 & PPD & $\begin{array}{l}\text { PD with } \\
\text { Dysphagia } \\
\text { complaint } \\
\mathrm{s} \\
\mathrm{n}=109\end{array}$ & $\begin{array}{l}\text { Compared the effect of } \\
\text { Surface NMES on } 3 \\
\text { groups } \\
\text { group1: received only } \\
\text { traditional dysphagia } \\
\text { therapy } \\
\text { group 2: received } \\
\text { traditional therapy + } \\
\text { surface electrical } \\
\text { stimulation at motor level } \\
\text { group 3: received } \\
\text { traditional dysphagia } \\
\text { therapy as well as surface } \\
\text { electrical stimulation at } \\
\text { sensory level } \\
\text { session: 15 sessions , } 1 \\
\text { session per day lasting for } \\
0.5 \text { hour }\end{array}$ & Submental region & $\begin{array}{l}\text { Videofluroscopic } \\
\text { study and Fiberoptic } \\
\text { Endoscopic } \\
\text { Evaluation } \\
\text { Swallowing } \\
\text { for temporal, spatial } \\
\text { and visoperceptual } \\
\text { variables before and } \\
\text { after treatment for } 3 \\
\text { different bolus } \\
\text { consistency(thin } \\
\text { liquid, thick liquid } \\
\text { and small bite } \\
\text { crackers) }\end{array}$ & 0 & $\begin{array}{l}\text { A } \\
\text { (Prospective } \\
\text { RCT) }\end{array}$ \\
\hline
\end{tabular}

Table 1: Author, year, research design, treatment protocol, electrode placement, outcome measures, results and evidence levels of the studies included for data extraction

Note: PPD - Pre-post-test design; 0 - no significant difference seen in the result 
The table clearly explains the research design used along with the total number of subjects, the method, the placement of the electrodes for NMES, subjective and objective instrumentation and the outcome parameters/measures. The result of the studies included depicts insignificant improvements or effect of the use of NMES on swallow or on dysphagia management in persons with PD. 2 out of 3 articles selected shows an evidence level A representing a well-designed randomised clinical trial (RCT), and one article was rated $\mathrm{B}$ on the evidence level representing a non-randomised control trial in accordance to the $\mathrm{ABC}$ rating scale [20].

Evidence documented by Baijens [2] was a Pioneer study in this arena without any existing literature and studied the effect of surface NMES on swallowing in persons with PD exhibiting dysphagia. They compared the effect on 10 subjects with mild- severe dysphagia with age-matched healthy controls. Both the groups performed 12 swallows each containing $10 \mathrm{cc}$ thin liquid barium boluses with a single session of surface NMES given simultaneously with VFSS in 3 electrode position. The outcome measures include timed variables (moment of opening - closing of glosso palatal junction, velopharyngeal junction, laryngeal vestibule and UES) as well as spatial variables (movement pattern of hyoid bone, pre swallow, anterior-posterior spill, lingual pumping, swallow hesitancy, piecemeal deglutition, delayed initiation of pharyngeal reflexes, post swallow oral residue, post swallow vallecular pooling, post swallow pyriform sinus pooling). The effect of electrical stimulation revealed insignificant effect for a majority of the variables except for the moment of opening closing of laryngeal vestibule duration and hyoid motion mainly due to single session and fixed stimulation variables which were not optimal for inducing any effects.

The same authors in 2013 [3] compared the effect of sensory NMES in 3 groups of persons with idiopathic PD. NMES therapy was given by various trained therapists. The maximum tolerated stimulation level resulting in maximum muscle contraction without a spasm was applied as motor level stimulation. The therapists raised the current intensity gradually until the first sensation of stimulation was felt by the patient in the sensory level stimulation. The results derived were not statistically significant probably due to inappropriately fixed stimulation variables. Moreover, oropharyngeal dysphagia in PD could be due to an impairment in the Central Nervous System and Peripheral Nervous System pathways of swallowing. Heinen, Spyer, Baijens and Boogart in 2012 [6] studied the Swallow Quality of Life in dysphagic patients with PD divided into three groups (A, B and C) with A receiving only traditional swallow therapy, B receiving only NMES and $\mathrm{C}$ receiving both NMES and standard therapy given by various trained therapists. The outcome measures when statistically analysed showed no significant treatment effect on SWALQOL, and no group difference was seen.

The strengths of the articles included were that the authors implemented a strict and homogenous inclusion criterion; the outcome values obtained were reconfirmed several times to correct for sensory adaptation. The RCTs were of adequate sample size, and the authors blinded judges for all measurements and rating. The inter and intra rater reliabilities were determined as well. The limitations leading to insignificant effect can be attributed to the inclusion of various airway protection manoeuvres, bolus modification, oro motor exercises and rehabilitative techniques in the traditional therapy as the actual effect of each method cannot be assessed and the outcome obtained is the effect of all the therapy technique used in unison. Moreover, no counterbalancing has been done between the groups to yield the actual impact of the methods used, and the long term effects of the therapy have not been documented. No other research has been carried out in the recent past paving the way to the current researchers to fill the existing gap in the evidence.

\section{Discussion}

The objectives of this systematic review were to collate, summarise and critically analyse the existing studies reporting the effect of NMES on swallowing in persons with PD exhibiting dysphagia. Among the limited studies retrieved from the databases, only three articles passed the data extraction stage. Two out of three articles selected to be reviewed qualified for level A evidence on further analysis. All studies followed a prospective pre-test post-test comparison design. The studies incorporating an experimental procedure which were conducted on PD and examined the effects of NMES on swallowing as a treatment technique were included. The articles were of high quality as they were controlled trials with assessors blinded, randomised samples which were adequately described, groups/ participants were compared at baseline on essential factors, the effect size and confidence intervals were reported and calculated, and most of the outcome measures were valid and reliable. In general, the majority of the studies reporting the use of NMES in dysphagia management with positive outcomes are readily available in individuals with post-stroke due to easy availability and referrals. Although NMES can be advantageous in PD due to its non-invasive nature, ease to use with reduced muscle fatigue not involving the active participation of the persons receiving it and clinician driven, Critical analysis of the finalised articles revealed that the studies were carried out by the same authors, suggesting lack of research on the topic proving the efficacy of the use of NMES as a dysphagia management approach in persons with $\mathrm{PD}$.

Lack of studies on dysphagia management using NMES in persons with PD can be attributed to its asymptomatic nature in the early stage of progression and low referrals by the healthcare professionals due to its degenerative condition. The three studies included have studied the effect of NMES only on patients with Idiopathic PD reporting dysphagia symptoms. Inclusion of Parkinson's Plus syndromes along with Persons with PD can give us more insight and differences in the reduction, as well as prevention of dysphagia symptom using NMES as a component of dysphagia management Inclusion of case studies in the review, could have shed more light on the benefits of using NMES on swallow function in PD. The studies included in this review did not include the persons diagnosed with dysphagia due to multiple aetiologies, including PD. Inclusion of PD as subjects in these studies reporting the efficacy of NMES in dysphagia does not shed light on the effectiveness isolated in PD. Hence general inference drawn is not particular to Persons with PD and cannot be concluded.

Sufficient evidence stating insignificant therapy effects could not be found due to a lack of well-designed studies with poor blinding criterion and Poor choice of assessment tools as well as the outcome variable. Inclusion of PD with mild - severe dysphagia in the studies reviewed can be a variable influencing the outcome of the studies as pathological mechanisms of chronic dysphagia in PD is complex and is comparatively different as it involves the coordination of the peripheral and central nervous pathways and many reports evidence intact swallow physiology in the early phase of PD.

A number of other variables affecting the efficacy of the studies documented in the review can be contributed to the placement of electrodes and the intensity and amplitude of the stimulation as it has been reported that physiology of motor and sensory mechanism of swallow in PD must be studied prior to the application on the affected muscle [22]. Multidimensional assessment of the therapeutic outcome is required. Measurement protocols must be standardised and based on the reproducible reliable rating of well-defined variables. Better quality of life scores could be attained along with a global assessment score and with increased therapeutic duration. Since PD is degenerative in nature, it is of utmost importance to give continuous therapy [19].Van Hoorens [23] review on the dysphagia management in PD, highlights on the use of medication, alternative approaches such as logopedic dysphagia 
treatment, EMST, VAST, swallow exercises as efficient, cost-effective and proves that these dysphagia management techniques focus on a broader spectrum of severity and long term outcomes when compared to NMES which has fewer shreds of evidence documented.

However, the current review implemented is one of the pioneering reviews as no review was reported to be found in the literature search previously in the domain despite including studies for a period of 20 years (between January 2000 to January 2020). The articles included being recent, calls attention for more scientific research to be done. The findings of the included evidence have provided important insights to the positive outcomes of effects of NMES such as shown in improved measures on the moment of opening-closing of laryngeal vestibule duration and hyoid motion [2] and significant therapy effects on Dysphagia Severity Scale [6] which further supports the fact that an appropriate number of sessions as well as understating the pathophysiological changes in the muscle functioning involving swallowing in each individuals reporting swallowing problems can show better progress in swallow function with the use of NMES as suggested by Jeri Logemann [12] in an invited editorial. Park et al [16] studied the effect of effortful swallow with NMES as an adjunct in patients with PD with dysphagia. their findings reported a significant effect, supported by increase in the horizontal and vertical movement of hyoid bone as recorded on VFSS. The authors concluded that the therapeutic techniques used effectively increased the hyoid bone movement and reduced aspiration. This study was not included in the review as it was done by the physical therapists and did not fit in the inclusion criteria which could have been an additional evidence supporting the positive effects of use of NMES in PD.

The literature on the use of NMES in individuals with dysphagia reports contradicting evidence and doesn't come to conclusive proof. Humbert [8],[9] reports reduced hyo- laryngeal elevation during electrical stimulation in normal individuals suggesting inefficient swallow physiology; however, Ludlow [13] argues that aspiration and pooling were significantly reduced in chronic dysphagia. All three studies included in the review documents no significant effect of the use of NMES in Persons with PD, however, researchers need to contribute more to prove the actual efficacy before considering it as ineffective as the existing studies do not provide sufficient information to conclude.

\section{Conclusions}

Among various articles reporting the use of NMES in dysphagia management, evidence reporting the use of NMES in PD is limited. This systematic review attempted to summarise, critically and qualitatively analyse the prevailing evidence for the benefit of NMES in dysphagia management in persons with PD. The existing research shows no significant effect of NMES on swallow function in dysphagic individuals with PD. The outcome of the current studies cannot be generalised across the population despite them showing no significant effects, there is a need for high-quality controlled trials (Clarke et al.). Additional research is needed to address other research questions such as at what point in the disease process or recovery NMES is more beneficial and whether optimal timing/treatment dosage may vary across stimulation parameters which could have affected the findings of the current studies included in the review.

There is lack of research and evidence along with several methodological flaws concerning the use of NMES in persons with PD which can be further studied by aspiring researchers, modifying the methodologies used in the previous studies.

Acknowledgements: We thank the Director - AIISH, for providing all the necessary resources and infrastructure for this study.

Funding: This study received no grant from any agency.
Conflict of interests: The authors declare that they have no conflict of interest.

\section{References}

1. Baijens, L. W., \& Speyer, R. (2009). Effects of therapy for dysphagia in Parkinson's disease: systematic review. Dysphagia, 24(1), 91-102.

2. Baijens, L. W., Speyer, R., Passos, V. L., Pilz, W., Roodenburg, N., \& Clavé, P. (2012). The effect of surface electrical stimulation on swallowing in dysphagic Parkinson patients. Dysphagia, 27(4), 528-537.

3. Baijens, L. W., Speyer, R., Passos, V. L., Pilz, W., van der Kruis, J., Haarmans, S., \& Desjardins-Rombouts, C. (2013). Surface electrical stimulation in dysphagic Parkinson patients: a randomized clinical trial. The Laryngoscope, 123(11), E38E44.

4. Clave, P., Terré, R. D., De Kraa, M., \& Serra, M. (2004). Approaching oropharyngeal dysphagia. Revista Espanola de Enfermedades Digestivas, 96(2), 119-131.

5. Ekberg, O., Hamdy, S., Woisard, V., Wuttge-Hannig, A., \& Ortega, P. (2002). Social and psychological burden of dysphagia: its impact on diagnosis and treatment. Dysphagia, 17(2), 139-146.

6. Heijnen, B. J., Speyer, R., Baijens, L. W. J., \&Bogaardt, H. C. A. (2012). Neuromuscular electrical stimulation versus traditional therapy in patients with Parkinson's disease and oropharyngeal dysphagia: effects on quality of life. Dysphagia, 27(3), 336-345.

7. Parkinson's Foundation (n.d.). Understanding Parkinson's: Statistics.

8. Humbert, I. A., Michou, E., MacRae, P. R., \& Crujido, L. (2012). Electrical stimulation and swallowing: how much do we know? Seminars in Speech and Language, 33(3), 203-216.

9. Humbert, I. A., Poletto, C. J., Saxon, K. G., Kearney, P. R., Crujido, L., Wright-Harp, W., Payne, J., Jeffries, N., Sonies, B. C., \& Ludlow, C. L. (2006). The effect of surface electrical stimulation on hyolaryngeal movement in normal individuals at rest and during swallowing. Journal of Applied Physiology, 101(6), 1657-1663.

10. Kwon, M., \& Lee, J. H. (2019). Oro-pharyngeal dysphagia in Parkinson's disease and related movement disorders. Journal of Movement Disorders, 12(3), 152-160.

11. Leopold, N. A., \& Kagel, M. C. (1996). Prepharyngeal dysphagia in Parkinson's disease. Dysphagia, 11(1), 14-22.

12. Logemann, J. A. (2007). The effects of VitalStim on clinical and research thinking in dysphagia. Dysphagia, 22(1), 11-12.

13. Ludlow, C. L. (2013). Electrical Stimulation Treatment. In R. Shaker, P. C. Belafsky, G. N. Postma, \& C. Easterling (Eds.), Principles of Deglutition: A Multidisciplinary Text for Swallowing and its Disorders (pp. 809-820). Springer.

14. Moher, D., Shamseer, L., Clarke, M., Ghersi, D., Liberati, A., Petticrew, M., Paul, S., Stewart, L. A., \& PRISMA-P Group (2015). Preferred reporting items for systematic review and meta-analysis protocols (PRISMA-P) 2015 statement. Systematic reviews, 4(1), 1-9.

15. Nilsson, H., Ekberg, O., Olsson, R., \& Hindfelt, B. (1996). Quantitative assessment of oral and pharyngeal function in Parkinson's disease. Dysphagia, 11(2), 144-150.

16. Park, J. S., Oh, D. H., Hwang, N. K., \& Lee, J. H. (2018). Effects of neuromuscular electrical stimulation in patients with Parkinson's disease and dysphagia: a randomized, single-blind, placebo-controlled trial. Neuro Rehabilitation, 42(4), 457-463.

17. Pflug, C., Bihler, M., Emich, K., Niessen, A., Nienstedt, J. C., Flügel, T., Koseki, J-C., Plaetke, R., Hidding, U., Gerloff, C., 
\& Buhmann, C. (2018). Critical dysphagia is common in Parkinson disease and occurs even in early stages: a prospective cohort study. Dysphagia, 33(1), 41-50.

18. Potulska, A., Friedman, A., Królicki, L., \& Spychala, A. (2003). Swallowing disorders in Parkinson's disease. Parkinsonism \& Related Disorders, 9(6), 349-353.

19. Sardi, S. P., Cedarbaum, J. M., \&Brundin, P. (2018). Targeted therapies for Parkinson's disease: from genetics to the clinic. Movement Disorders, 33(5), 684-696.

20. Siwek, J., Gourlay, M. L., Slawson, D. C., \& Shaughnessy, A. F. (2002). How to write an evidence-based clinical review article. American Family Physician, 65(2), 251-258.
21. Suttrup, I., \&Warnecke, T. (2016). Dysphagia in Parkinson's disease. Dysphagia, 31(1), 24-32.

22. Tjaden, K. (2008). Speech and swallowing in Parkinson's disease. Topics in Geriatric Rehabilitation, 24(2), 115-126.

23. Van Hooren, M. R. A., Baijens, L. W. J., Voskuilen, S., Oosterloo, M., \& Kremer, B. (2014). Treatment effects for dysphagia in Parkinson's disease: a systematic review. Parkinsonism \& Related Disorders, 20(8), 800-807.

24. Won, J. H., Byun, S. J., Oh, B. M., Kim, H. J., Park, S. J., \&Seo, H. G. (2020). Pneumonia risk and its associated factors in Parkinson's disease: A National Database Study. Journal of the Neurological Sciences, 415:116949.

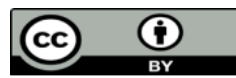

This work is licensed under Creative Commons Attribution 4.0 License

To Submit Your Article Click Here: Submit Manuscript

DOI: $10.31579 / 2690-4861 / 105$
Ready to submit your research? Choose Auctores and benefit from:

* fast, convenient online submission

* rigorous peer review by experienced research in your field

* rapid publication on acceptance

* authors retain copyrights

* unique DOI for all articles

* immediate, unrestricted online access

At Auctores, research is always in progress.

Learn more www.auctoresonline.org/journals/international-journal-ofclinical-case-reports-and-reviews 